\title{
Improving an Association Rule Based Classifier
}

\author{
Bing Liu, Yiming Ma, and Ching Kian Wong \\ School of Computing \\ National University of Singapore \\ 3 Science Drive 2, Singapore 117543 \\ \{liub, maym, wongck\} @comp.nus.edu.sg
}

\begin{abstract}
Existing classification algorithms in machine learning mainly use heuristic search to find a subset of regularities in data for classification. In the past few years, extensive research was done in the database community on learning rules using exhaustive search under the name of association rule mining. Although the whole set of rules may not be used directly for accurate classification, effective classifiers have been built using the rules. This paper aims to improve such an exhaustive search based classification system CBA (Classification Based on Associations). The main strength of this system is that it is able to use the most accurate rules for classification. However, it also has weaknesses. This paper proposes two new techniques to deal with these weaknesses. This results in remarkably accurate classifiers. Experiments on a set of 34 benchmark datasets show that on average the new techniques reduce the error of CBA by $17 \%$ and is superior to CBA on 26 of the 34 datasets. They reduce the error of $\mathrm{C} 4.5$ by $19 \%$, and improve performance on 29 datasets. Similar good results are also achieved against RIPPER, LB and a Naïve-Bayes classifier.
\end{abstract}

\section{Introduction}

Building effective classification systems is one of the central tasks of data mining. Past research has produced many techniques and systems (e.g., C4.5 [10], and RIPPER [3]). The existing techniques are, however, largely based on heuristic/greedy search. They aim to find only a subset of the regularities that exist in data to form a classifier.

In the past few years, the database community studied the problem of rule mining extensively under the name of association rule mining [1]. The study there is focused on using exhaustive search to find all rules in data that satisfy the user-specified minimum support (minsup) and minimum confidence (minconf) constraints.

Although the complete set of rules may not be directly used for accurate classification, effective and efficient classifiers have been built using the rules, e.g., CBA [7], LB [8] and CAEP [4]. The major strength of such systems is that they are able to use the most accurate rules for classification. This explains their good results in general. However, they also have some weaknesses, inherited from association rule mining.

- Traditional association rule mining uses only a single minsup in rule generation, which is inadequate for unbalanced class distribution (this will be clear later).

- Classification data often contains a huge number of rules, which may cause combinatorial explosion. For many datasets, the rule generator is unable to generate rules with many conditions, while such rules may be important for classification.

D.A. Zighed, J. Komorowski, and J. Zytkow (Eds.): PKDD 2000, LNAI 1910, pp. 504-509, 2000.

(c) Springer-Verlag Berlin Heidelberg 2000 
This paper aims to improve the CBA system (Classification Based on Associations) by dealing directly with the above two problems. It tackles the first problem by using multiple class minsups in rule generation (i.e., each class is assigned a different minsup), rather than using only a single minsup as in CBA. This results in a new system called msCBA. Experiments on a set of 34 benchmark problems show that on average msCBA achieves lower error rate than CBA, C4.5 (tree and rules), and a Naïve-Bayse classifier (NB), LB and RIPPER (CAEP is not available for comparison).

The second problem is more difficult to deal with directly as it is caused by exponential growth of the number of rules. We deal with it indirectly. We try to find another classification technique that is able to help when some rules from msCBA are not accurate. The decision tree method is a clear choice because decision trees often go very deep, i.e., using many conditions. We then propose a technique to combine msCBA with the decision tree method as in C4.5. The basic idea is to use the rules of msCBA to segment the training data and then select the classifier that has the lowest error rate on each segment to classify the future cases falling into the segment. This composite method results in remarkably accurate classifiers.

\section{Association Rule Mining for Classification}

Association rule mining is stated as follows [1]: Let $I=\left\{i_{1}, i_{2}, \ldots, i_{m}\right\}$ be a set of items. Let $D$ be a set of transactions (the dataset), where each transaction $d$ (a data record) is a set of items such that $d \subseteq I$. An association rule is an implication of the form, $X \rightarrow$ $Y$, where $X \subset I, Y \subset I$, and $X \cap Y=\varnothing$. The rule $X \rightarrow Y$ holds in the transaction set $D$ with confidence $c$ if $c \%$ of transactions in $D$ that support $X$ also support $Y$. The rule has support $s$ in $D$ if $s \%$ of the transactions in $D$ contains $X \cup Y$.

Given a set of transactions $D$ (the dataset), the problem of mining association rules is to discover all rules that have support and confidence greater than the user-specified minimum support (called minsup) and minimum confidence (called minconf). An efficient algorithm for mining association rules is the Apriori algorithm [1].

Mining association rules for classification: The Apriori algorithm finds association rules in a transaction data of items. A classification dataset, however, is normally in the form of a relational table. Each data record is also labeled with a class. The table data can be converted to transaction data as follows: We first discretize each continuous attribute into intervals (see e.g., [5] [6] on discretization algorithms). After discretization, we can transform each data record to a set of (attribute, value) pairs and a class label, which is in the transaction form. A (attribute, value) pair is an item.

For classification, we only need to generate rules of the form $X \rightarrow c_{i}$, where $c_{i}$ is a possible class. We call such rules the class association rules (CARs). It is easy to modify the Apriori algorithm to generate CARs. We will not discuss it here (see [7]).

\section{Classifier Building in CBA}

After all rules (CARs) are found, a classifier is built using the rules. In CBA, a set of high confidence rules is selected from CARs to form a classifier (this method is also used in msCBA). The selection of rules is based on a total order defined on the rules. 
Definition: Given two rules, $r_{i}$ and $r_{j}, r_{i} \succ r_{j}$ (also called $r_{i}$ precedes $r_{j}$ or $r_{i}$ has a higher precedence than $r_{j}$ ), if the confidence of $r_{i}$ is greater than that of $r_{j}$, or if their confidences are the same, but the support of $r_{i}$ is greater than that of $r_{i}$, or if both the confidences and supports of $r_{i}$ and $r_{j}$ are the same, but $r_{i}$ is generated earlier than $r_{j}$;

Let $R$ be the set of CARs, and $D$ the training data. The basic idea of the classifierbuilding algorithm in CBA is to choose a set of high precedence rules in $R$ to cover $D$. A CBA classifier is of the form:

$$
\left\langle r_{1}, r_{2}, \ldots, r_{n}, \text { default_class }\right\rangle .
$$

where $r_{i} \in R, r_{a} \succ r_{b}$ if $b>a$. In classifying an unseen case, the first rule that satisfies the case classifies it. If no rule applies, the default class is used. A simple version of the algorithm for building such a classifier is given in Figure 1. [7] presents an efficient implementation of the algorithm. It makes at most two passes over the data.

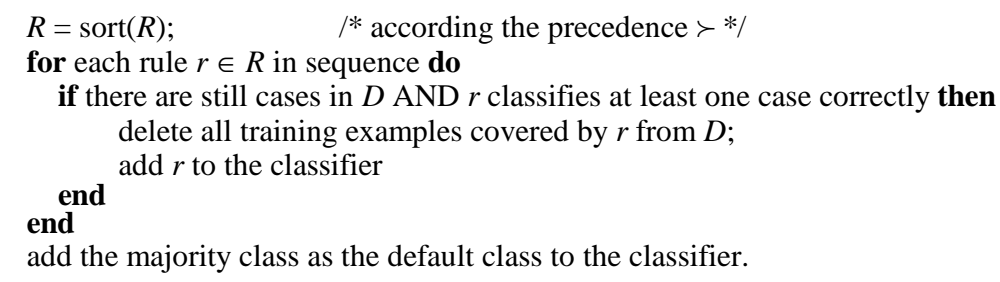

Fig. 1. A simple classifier-building algorithm

\section{Improving CBA}

\subsection{Using Multiple Minimum Class Support}

The most important parameter in association rule mining is the minsup. It controls how many rules and what kinds of rules are generated. The CBA system follows the classic association rule model and uses a single minsup in its rule generation. We argue that this is inadequate for mining of CARs because many practical classification datasets have uneven class frequency distributions. If we set the minsup value too high, we may not find sufficient rules of infrequent classes. If we set the minsup value too low, we will find many useless and over-fitting rules for frequent classes. To solve the problems, msCBA adopts the following (multiple minimum class supports):

minsup $_{\mathrm{i}}$ : For each class $c_{i}$, a different minimum class support is assigned. The user only gives a total minsup, denoted by $t_{-}$minsup, which is distributed to each class:

$$
\operatorname{minsup}_{\mathrm{i}}=\mathrm{t} \_ \text {minsup } \times \text { freqDistr }\left(\mathrm{c}_{\mathrm{i}}\right) .
$$

The formula gives frequent classes higher minsups and infrequent classes lower minsups. This ensures that we will generate sufficient rules for infrequent classes and will not produce too many over-fitting rules for frequent classes.

\subsection{Seeking Help from Other Techniques}

As we mentioned earlier, for many datasets, the rule generator is unable to generate rules with many conditions (i.e., long rules) due to combinatorial explosion. When 
such long rules are important for classification, our classifiers suffer. Here, we propose a combination technique. The aim is to combine msCBA with a method that is able to find long rules. Clearly, the decision tree method is a natural choice because decision trees often go very deep, i.e., using many conditions. In our implementation, we also include the Naïve-Bayes method (NB) as NB comes free from msCBA (the probabilities needed by NB are all contained in the 1-condition rules of msCBA).

The proposed combination method is based on the competition of different classifiers on different segments of the training data. The key idea is to use one classifier to segment the training data, and then choose the best classifier to classify each segment.

Let $A$ be the classifier built by msCBA, $T$ be the decision tree built by $\mathrm{C} 4.5$, and $N$ be the Naïve-Bayse classifier. We use the rules in $A$ to segment the data. For the set of training examples covered by a rule $r_{i}$ in $A$, we choose the classifier that has the lowest error on the set of examples to replace $r_{i}$. That is, if $r_{i}$ has the lowest error, we keep $r_{i}$. If $T$ has the lowest error, we use $T$ to replace $r_{i}$. If $r_{i}$ is replaced by $T$, then in testing when a test case satisfies the conditions of $r_{i}$, it is classified by $T$ instead of $r_{i}$. The same applies to $N$. The algorithm is given in Figure 2.

From line 3-6, we compute the number of errors made by $r_{i}, T$, and $N$ on the training examples covered by each $r_{i}$. Error ${ }_{i}$, Error $_{T i}$ and Error $_{N i}$ are initialized to 0. From line 9-11, we use $T$ (or $N$ ) to replace $r_{i}$ if $T$ ( or $N$ ) results in fewer errors on the training examples covered by $r_{i}$. $X \rightarrow$ (use $T$ ) means that in testing if a test case satisfies $X$ (the conditions of $\left.r_{i}\right), T$ will be used to classify the case.

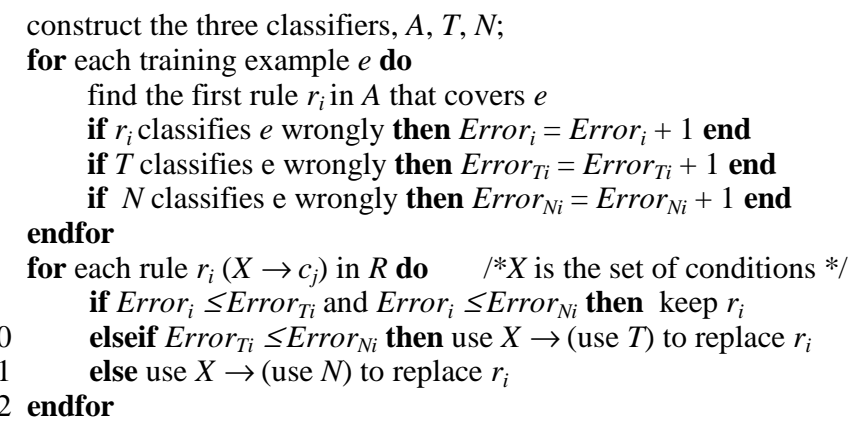

Fig. 2. The combination algorithm

\section{Experiments}

We now compare the classifiers built by msCBA, CBA, C4.5 (tree and rules, Release 8), RIPPER, NB, LB, and various combinations of msCBA, C4.5 and NB. The evaluations are done on 34 datasets from UCI ML Repository [9]. We also used Boosted C4.5 (the code is obtained from Zijian Zheng [11]) in our comparison. We ran all the systems using their default settings. We could not compare with existing classifier combination methods as we were unable to obtain the systems.

In all the experiments with msCBA, minconf is set to 50\%. For t_minsup, from our experience, once $t \_$minsup is lowered to $1-2 \%$, the classifier built is already very accurate. In the experiment results reported below, we set $t_{-}$minsup to $1 \%$. 

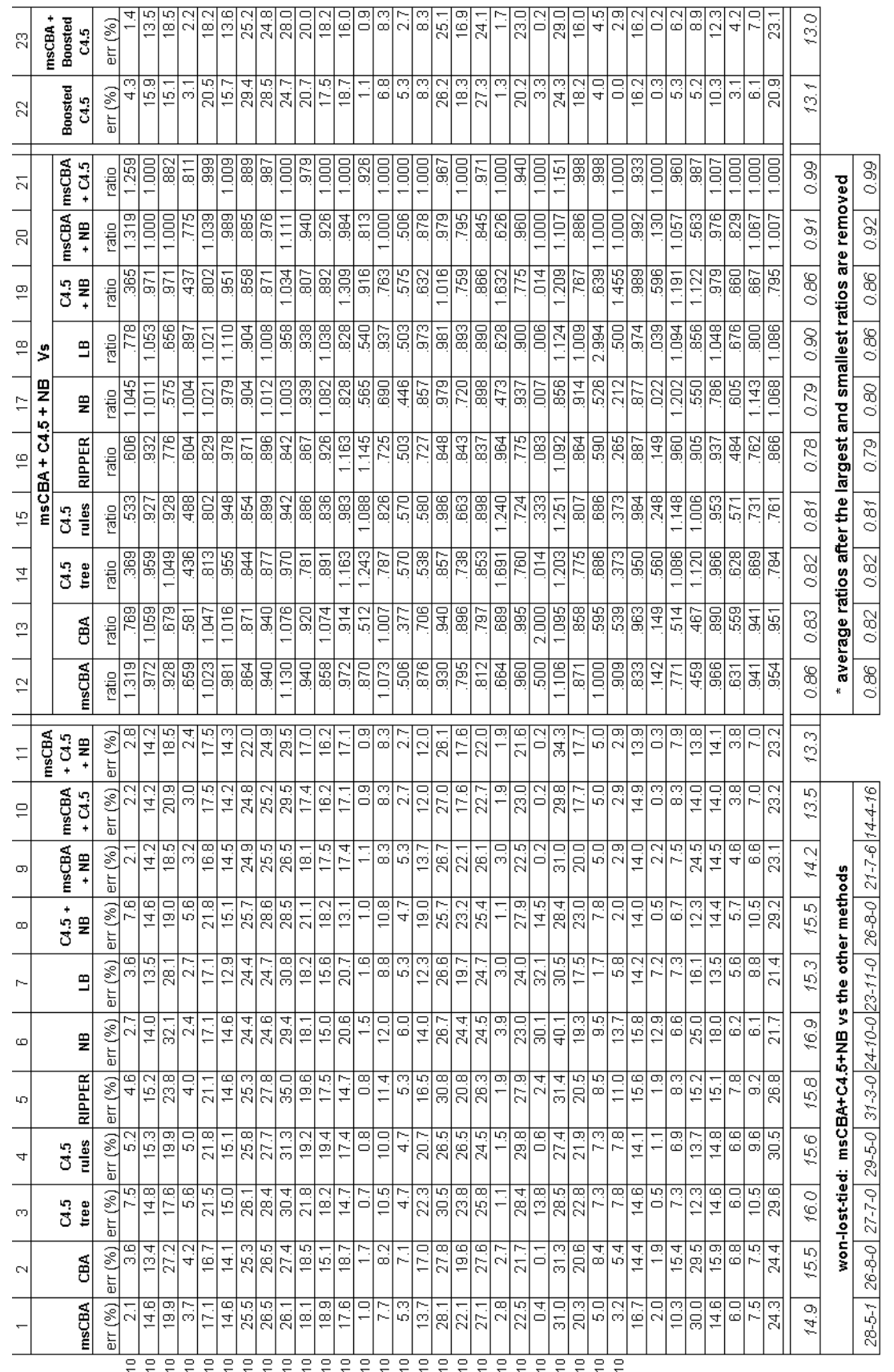

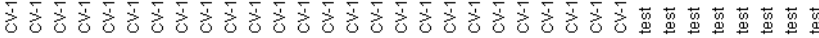

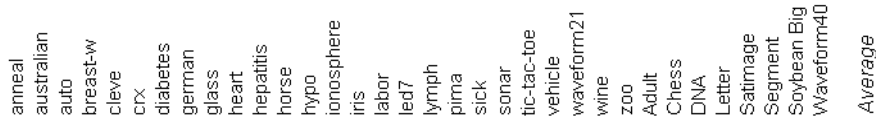


Experiment results are shown in Table 1. The error rates on the first 26 datasets are obtained from 10-fold cross-validation, while on the last 8 datasets they are obtained from the test sets. All the composite methods involving C4.5 uses C4.5 tree.

Error rate comparison: For each dataset, column 1-11 show the error rates of msCBA, CBA, C4.5 tree, C4.5rules, RIPPER, NB, LB, C4.5+NB (C4.5 tree combinedwith $\mathrm{NB}$ ), msCBA+NB, msCBA+C4.5 and msCBA+C4.5+NB respectively. Column 12-21 show the ratios of the error rate of msCBA+C4.5+NB vs. the other methods. From the table we see that on average the error rate of msCBA is lower than every other individual method. It is also clear that over the 34 datasets, the composite methods are superior to individual methods. $\mathrm{msCBA}+\mathrm{C} 4.5+\mathrm{NB}$ gives the lowest error rate on average. It reduces the error of $\mathrm{C} 4.5$ tree (or rules) by $18 \%$ (or 19\%) on average, and its won-lost-tied record against C4.5 tree (or rules) is 27-7-0 (or 29-5-0). It reduces the error of msCBA by $14 \%$, and its won-lost-tied record against msCBA is 28-5-1. Similar good results are also achieved against CBA, RIPPER, NB and LB.

$\mathrm{msCBA}+\mathrm{C} 4.5$ and msCBA+C4.5+NB have similar performances. This confirms our intuition that msCBA's weakness is overcome by deep trees of C4.5. Column 22 and 23 show the error rates of boosted $\mathrm{C} 4.5$ and msCBA+boostedC4.5. We see that msCBA+C4.5+NB's results are comparable to boosted C4.5, and its won-lost-tied record against boosted $\mathrm{C} 4.5$ is $18-15-1$. Since boosted $\mathrm{C} 4.5$ is regarded as one of the best classifiers, we can say that msCBA+C4.5+NB is also among the best.

\section{Conclusion}

This paper aims to improve an exhaustive search based classification system CBA. It first identified two weaknesses of the system, and it then proposed two new techniques to deal with the problems. The techniques produce markedly better classifiers.

\section{References}

1. Agrawal, R. \& Srikant, R. 1994. Fast algorithms for mining association rules. VLDB-94.

2. Chan, P. \& Stolfo, J. S. 1993. Experiments on multistrategy learning by meta-learning. Proc. Second Intl. Conf. Info. Know. Manag., 314-323.

3. Cohen, W. 1995. Fast effective rule induction. ICML-95.

4. Dong, G., Zhang, X. Wong, L. Li, J. 1999. CAEP: classification by aggregating emerging patterns. Discovery-Science-99.

5. Fayyad, U. \& Irani, K. 1993. Multi-interval discretization of continuous-valued attributes for classification learning. IJCAI-93, 1022-1027.

6. Kohavi, R., John, G., Long, R., Manley, D., \& Pfleger, K. 1994. MLC++: a machinelearning library in C++. Tools with artificial intelligence, 740-743.

7. Liu, B., Hsu, W. \& Ma, Y. 1998. Integrating classification and association rule mining. KDD-98.

8. Meretkis, D. \& Wuthrich, B. 1999. Extending naïve bayes classifiers using long itemsets. KDD-99.

9. Merz, C. J. \& Murphy, P. 1996. UCI repository of machine learning database. [http://www.cs.uci.edu/ mlearn].

10. Quinlan, J. R. 1992. C4.5: program for machine learning. Morgan Kaufmann.

11. Zheng, Z. and Webb, G. 1999. Stochastic attribute selection committees with multiple boosting: Learning more accurate and more stable classifier committees. PAKDD-99. 